\title{
HARDY'S DISCRETE INEQUALITY
}

\author{
G. D. Handley, J. J. Koliha AND J. PeČARIĆ
}

Abstract. Copson's extension of Hardy's discrete inequality has been generalised in different directions by Hwang, Hwang-Yang and Pachpatte. In this paper we obtain inequalities which subsume and extend results of Pachpatte (Libertas Math. 14 (1994), 151-157) and Hwang-Yang (Tamkang J. Math. 27 (1996), 125-132).

Mathematics subject classification (2000): 26D15.

Key words and phrases: Hardy's discrete inequality.

\section{REFERENCES}

[1] E. T. Copson, Note on series of positive terms, J. London Math. Soc. 3 (1928), 49-51.

[2] E. B. ElLiotT, A simple exposition of some recently proved facts as to convergency, J. London Math. Soc., 1 (1926), 93-96.

[3] G. H. Hardy, J. E. Littlewood And G. Polya, Inequalities, Cambridge University Press, Cambridge, 1934.

[4] DaH-YAn Hwang, A many variable generalization of the discrete Hardy's inequality, Tamkang J. Math. 27 (1996), 125-132.

[5] Dah-Yan Hwang And Gou-Sheng Yang, Note on discrete Hardy's inequality, Tamkang J. Math. 21 (1990), 333-336.

[6] D. S. MitrinOvić, J. E. PEČARIĆ AND A. M. FINK, Inequalities involving functions and their integrals and derivatives, Kluwer Academic, Dordrecht, 1991.

[7] B. G. PachPatTe, On Hardy-type discrete inequalities, Libertas Math. 14 (1994), 151-157. 